\title{
Relationship Between Breeding Systems and Resistance to Mercury in Euplotes crassus (Ciliophora: Hypotrichida)*
}

\author{
F. DINI** \\ Department of Zoology, University of Toronto, Toronto, Ontario, Canada M5S 1A1 \\ and \\ Istituto di Zoologia, Università di Pisa, 56100 Pisa, Italy
}

\begin{abstract}
A toxicity bioassay was used to measure the median tolerance limit to mercuric ion of different stocks of the marine hypotrichous ciliate Euplotes crassus. The stocks were representatives of local populations diversified in their breeding systems (inbreeding or outbreeding). The results show a greater tolerance to mercury in outbreeders. Genetic analysis of interstock tolerance-differences indicates no simple genetic basis. An interpretation is presented which suggests that the different degrees of mercury tolerance revealed by the representatives of inbreeding and outbreeding populations could depend on their peculiar genetic organizations. Such peculiar genetic organizations might be the product of the distinct ecogenetic strategies followed by the populations to which the stocks belong.
\end{abstract}

\section{INTRODUCTION}

Excess toxic substances are often introduced into ecosystems as by-products of industrial processes. The sudden changes in concentration of toxicants usually subject organisms to severe stress. There is ample experimental evidence that different species - as well as different populations belonging to the same species - may vary, sometimes widely, as far as the tolerance to a particular toxic agent is concerned (e.g. reviews by Ford, 1971 and Cairns, 1974). Once these observations become established, the investigation of the mechanisms responsible for the differential susceptibility of the organisms, and the factors affecting their ability to withstand varied toxicant concentrations in the environment, become the major tasks for the biologist. The system of breeding (the degree of inbreeding or outbreeding) followed by an organism may be an impor-

- This work was partially accomplished at the Ramsay Wright Zoological Laboratories, University of Toronto, Canada, with the support of a NATO Fellowship awarded to F. Dini through the Italian National Research Council (CNR)

- Present address: Instituto di Zoologia, Università di Pisa, Via A. Volta 4, 56100 Pisa, Italy tant factor determining the degree of resistance to environmental stress (Nyberg, 1974)

The object of the present study was to establish whether a relationship exists between breeding systems and resistance to severe stress caused by mercury. As is well known, mercury plays an important role in water pollution.

The cosmopolitan marine ciliate Euplotes crassus recently found to comprise populations committed to either inbreeding or outbreeding (Luporini and Dini, 1977) - was used as a model system. The overall morphological features of the stocks used in this investigation conform fairly well to the general description of the classical morphospecies E. crassus. Yet, these stocks consist of at least two sets of mating types between which gene flow does not occur; this indicates that they constitute two distinct biological species. However, the fact that related biological species cannot yet be identified reliably leads me to group both aforementioned species in the same classical, typologically-defined species - E. crassus. The observations to be reported indicate that mercury is highly toxic, even in trace amounts, and indicate a higher tolerance to mercury in outbreeding than inbreeding populations of E. crassus. 


\section{MATERIALS AND METHODS}

\section{Natural Sources}

Of the 5 stocks of Euplotes crassus used, $\mathrm{G}_{\mathrm{I}}, \mathrm{G}_{\mathrm{VT1}}$, and $\mathrm{G}_{11}$ were derived from collections at the same locality, whereas $\mathrm{PB}_{1}$ and $\mathrm{ST}$ were collected from different locations, both geographically very far from the former The locations from which the stocks were collected are listed in Table 1. gation was attained at about the same time, following an immature period which was short compared with the immature period of the $A^{-}$stocks (Luporini and Dini, 1977; unpubl. observations). The duration of the other stages of the life cycle was also relatively short, and so was the total life cycle (Dini and Bertini, 1978; unpubl, observations). Moreover, several indications led Luporini and Dini (1977) to argue that the occurrence of conjugation was largely a laboratory phenomenon, suggesting that autogamy was the more

Table 1. Euplotes crassus. Geographic location, breeding pattern, stock designation, and mating relations among the stocks used. Percentage of mating was scored by observation of living cells under a stereomicroscope $(20 \times)_{i}++++$ roughly $70 \%$ of the cells engaged in pairing; ++ roughly $20 \%$; roughly $10 \% ;-0 \%$

\begin{tabular}{|c|c|c|c|c|c|c|c|}
\hline \multirow[t]{2}{*}{ Geographic location } & \multirow[t]{2}{*}{ Breeding pattern } & \multicolumn{6}{|c|}{ Mating relations } \\
\hline & & Stock & $\mathrm{G}_{\mathrm{I}}$ & $\mathrm{G}_{V T I}$ & $\mathrm{G}_{11}$ & $\mathrm{~PB}_{1}$ & ST \\
\hline Gesira/Somalia (Indian Ocean) & Inbreeding & $\begin{array}{l}\mathrm{G}_{\mathrm{I}} \\
\mathrm{G}_{\mathrm{VII}}\end{array}$ & & ++ & $\begin{array}{l}+ \\
++\end{array}$ & $\begin{array}{l}+ \\
+\end{array}$ & $\overline{-}$ \\
\hline $\begin{array}{l}\text { Gesira/Somalia (Indian Ocean) } \\
\text { Piombino/Italy (Tyrrhenian Sea) } \\
\text { S. Terenzo/Italy (Ligurian Sea) }\end{array}$ & Outbreeding & $\begin{array}{l}\mathrm{G}_{11} \\
\mathrm{~PB}_{1} \\
\mathrm{ST}\end{array}$ & & & & +++ & - \\
\hline
\end{tabular}

\section{Breeding Features}

The Stocks $\mathrm{G}_{11}, \mathrm{~PB}_{1}$, and ST (hereafter referred to as 'non-autogamous, $A^{-}$, stocks') reproduced sexually by conjugation only (a process of reciprocal fertilization occurring between temporarily paired cells ordinarily of complementary mating type). They were chosen as the best characterized, healthy-growing representatives of local populations whose overall features speak for highly outbreeding patterns (Sonneborn, 1957; Nanney, 1980): long periods of sexual immaturity, maturity, senility, and a long life cycle; cross conjugation (interclonal conjugation) during maturity, but cross conjugation and selfing (intraclonal conjugation due to assortment of the altermative phenotypes of heterozygotes at the $m t$ locus) during senility; a system of multiple interbreeding mating types controlled by one-locus multiple alleles (unpubl. observations) according to the model proposed by Heckmann (1963). All these features, except selfing, concur to favour mating between unrelated partners in nature. If a suitable unrelated partner is not found, eventually selfing may occur; it serves to initiate a new life cycle. In the absence of fertilization death would occur.

The Stocks $G_{1}$ and $G_{\text {VII }}$ (referred to as autogamous, $\mathrm{A}^{+}$, stocks' in this paper) were representatives of populations in which fertilization occurred either by conjugation or by autogamy (a process of internal fertilization occurring in single, i. e. unpaired cells and representing the closest form of inbreeding; Luporini and Dini, 1977). The ability to undergo autogamy or conju- likely form of fertilization pursued by $\mathrm{A}^{+}$stocks in nature. All these features, plus the considerable nonviability following conjugation (Luporini and Dini, 1977), point toward an extremely inbreeding way of life (Sonneborn, 1957; Nanney, 1980). Of interest to this investigation are the genetic consequences of autogamy in Euplotes crassus. When the 2 unlinked loci a (autogamy) and $m$ t (mating type) were considered, the appearance of recombinants in heterozygous $\mathrm{A}^{+}$stocks after having passed through many successive autogamies was an extremely rare event (Luporini and Dini, 1977; Dini and Luporini, 1980). Hence it can be inferred that the $\mathrm{A}^{+}$stocks largely maintained their original genotypes characterized by a certain degree of heterozygosity (Luporini and Dini, 1977) - an inference confirmed by the present investigation, at least with respect to Stock $G_{\text {vil }}$.

\section{Breeding Relations}

$G_{I}, G_{V I I}, G_{11}$, and $P B_{1}$ were conspecific stocks each belonging to a different mating type. This statement was based on the results of mating tests (Table 1) and breeding analyses (taking as marker genes those determining autogamy (a) and/or mating type ( $m t)$ ) which indicate that all stocks were potentially able to exchange genes with each other to a greater or lesser degree (Luporini and Dini, 1977, unpubl. results). In combinations between the $A^{+}\left(G_{I}\right.$ and $\left.G_{V I I}\right)$ and $A^{-}\left(G_{11}\right.$ and $\left.P B_{1}\right)$ stocks feeble mating reactions, several 
induced homotypic pairs (between cells of the same mating type), and low viability rates after conjugation usually occurred, particularly when the $\mathrm{PB}_{1}$ stock was involved. The inherent low mating reactivity of the $\mathrm{A}^{+}$ stocks (Luporini and Dini, 1977), and a nucleo-cytoplasmic or genomic incompatibility between the two types of stock might be the major causes of the phenomena observed. Even though autogamous and non-autogamous populations displayed signs of reproductive isolation from each other, the tests for gene flow indicated that the threshold of an irreversible evolutionary divergence had not yet been passed.

Mating never occurred when the $A^{-}$Stock ST was repeatedly mixed with any of the foregoing set of stocks. Yet, it was able to mate with other stocks in mixtures performed on the same day. On the basis of the mating tests, it may be argued that Stock ST belongs to a different biological species.

\section{Cultivation Methods}

The standard procedure used in culturing stocks has already been described (Luporini and Dini, 1977). Following Heckmann (1963), stocks as well as their clonal cultures, were grown in Erd-Schreiber seawater medium inoculated with the alga Dunaliella salina, except that artificial seawater was used. The artificial seawater was prepared by dissolving Marine-Tropic New salt (Euraquarium s.p.a., Bologna) in slightly acidic, double-distilled water. It was always filtered and pasteurized at $85^{\circ} \mathrm{C}$ before use. Seawater parameters during the study were: $\mathrm{pH}=8.2 \pm 0.1$; salinity $=31.4 \pm 0.2 \% \mathrm{~S}$, dissolved oxygen from 7.1 to $8.5 \mathrm{mg}$ $1^{-1}$.

\section{Synchronization of Experimental Cells}

In order to perform experiments with cells of similar age from the same as well as from different stocks, new life-cycle stages of all the stocks used were contemporaneously initiated by autogamy or conjugation. (Age in ciliates is usually defined in terms of the number of divisions the organism has undergone since its last sexual process.) Clones of both $\mathrm{A}^{+}$stocks were established from exautogamous individuals taken from the original stock cultures. $A^{-}$stocks were cloned starting from exconjugant cells. They derived from selfing pairs spontaneously occurring in the original $\mathrm{PB}_{1}$ and ST stock cultures (heterozygous at the $m t$ locus). Selfing did not occur in Stock $\mathrm{G}_{11}$ because it was homozygous at the $m t$ locus. In this case, following the procedure employed by Nobili (1967), exconjugants were obtained from homotypic pairs induced by mixing Stock $\mathrm{G}_{11}$ with a long-maintained laboratory stock belonging to a different biological species and marked by small cell size. Only vigorously growing clones were retained, and they were maintained in active log-phase grow th by being fed excessive food at $20^{\circ}-21^{\circ} \mathrm{C}$. Under such conditions, the generation time for all the clones retained was approximately $14 \mathrm{~h}$. Because the generation times were closely similar, it could be argued that clones from $\mathrm{A}^{+}$and $\mathrm{A}^{-}$stocks were not populations of synchronously aging cells, since the two types of stock differed in their life spans. However, this difference may be considered immaterial because all clones were used before they overcame the sexual immaturity period. Moreover, Nyberg (1978) showed that older cells of the ciliate Paramecium tetraurelia are not necessarily more susceptible to environmental stress, as is often assumed.

The specimens employed in the experiments were also synchronized as to their cell-cycle stage. Since the experimental design was to test clones from the different stocks together on the same day, a group of cells in the late stage of division (easily recognized by the familiar peanut profile) was picked out of a log-phase clonal culture of each stock with a braking pipette and separately transferred into fresh culture medium diluted by the addition of an equal volume of seawater. The selection of each group of staged cells required a few minutes and was accomplished $1 \mathrm{~h}$ before its employment in the experiment. This time span was long enough to permit the morphogenetic events which culminate in successful cell division. The order of the experimental employment of the different cell groups followed the order of their isolation. In this way, owing to the similarity of the generation times, tested specimens from the same as well as from different clones were all synchronized in the early stage of the cell cycle.

\section{Bioassay Procedure}

Mercuric chloride of analytical quality (Fisher Scientific Company) was used to prepare a $10^{-3} \mathrm{M}$ solution in artificial seawater. The procedure employed by Nyberg $(1974,1975)$ for heavy metal tolerance tests in freshwater ciliates was followed with several modifications. An appropriate amount of $10^{-3} \mathrm{M} \mathrm{HgCl}_{2}$ was diluted with seawater to produce the test solution of the highest concentration used $(1.26 \mu \mathrm{M})$. The test solutions of lower concentration were made by consecutively diluting the next higher concentration with $20.9 \%$ seawater. In this way, the concentration range applied included a series of steps in concentration equally spaced on the log scale, in particular: roughly $1.26,1.0,0.80,0.63,0.40,0.32,0.25,0.20,0.16$, and $0.13 \mu \mathrm{M}$ mercuric ion. (One micromole $1^{-1}$ equals 0.2 
$\mathrm{mg}^{-1} \mathrm{Hg}^{++}$). For each stock tested, a different set of consecutive steps in concentration of the foregoing series was ordinarily used. In all cases the set employed covered the whole range of kills from zero to $100 \%$. The actual concentration of the metal ion in the solutions was not verified. Thus, it is only the 'nominal' rather than actual concentration. In view of the volatilization and surface adsorption phenomena occurring with respect to mercury (Glickstein, 1979), the abovementioned concentrations represent overestimations. The $\mathrm{pH}$ of the test solutions did not appreciatively differ from that of the seawater before addition of the metal ion. Just before the series of clones from different stocks were run through a mercury tolerance bioassay, new test solutions were prepared using the same beakers as containers. The same series of clones was run various times on successive days.

In testing the resistance to mercury three single specimens showing normal swimming behaviour were picked up with a braking pipette from a group of synchronized cells and transferred to each $0.5 \mathrm{ml}$ test solution dispensed into a well of a three-spot hemispheric depression slide. (Throughout this investigation, the same wells for the same test solutions were used.) Three replicates of each test concentration were always made, so that the toxic effect of mercury was assessed on a total of 9 specimens for each concentration at each run of each clone. The specimens were transferred into the test solutions starting with the controls and continuing towards the highest concentrations. (Each control well contained $0.5 \mathrm{ml}$ seawater identical to that in the experimental wells, except that it lacked the metal ion.) The amount of medium carried over with the cells at each transfer was less than one hundredth of the volume in a depression $(0.5 \mathrm{ml})$. The resulting dilution of the test solutions was, however, counterbalanced by the medium evaporation (about $4 \%$ ) occurring during the exposure time of cells to mercury. The 3 depression slides containing the cells were incubated inside moist chambers at $20^{\circ}-21^{\circ} \mathrm{C}$ in the dark. After $24 \pm 1 \mathrm{~h}$ the wells were checked and scored for surviving cells. Cells unable to swim or creep on the bottom of the well were regarded dead
There was variability in survival responses of the cells to a given mercury concentration among the runs of the same clone. Since such variability appeared to be distributed normally I proceeded to pool the data obtained at each concentration in each run over all runs. From the pooled survival responses at various concentrations the 24- $h$ median tolerance limit of the clone was estimated by probit analysis following Finney (1964). The median tolerance limit is an estimate of the median lethal concentration $\left(\mathrm{LC}_{50}\right)$, that is, the mercury concentration eliciting mortality in $50 \%$ of the test organisms.

\section{RESULTS}

\section{Mercury Tolerance of the Stocks}

The median tolerance limits of the clones bioassayed for each of the 5 stocks of Euplotes crassus are reported in Table 2. The values are given in $\mu \mathrm{M}^{-1} \mathrm{Hg}^{++}$. In addition to single clone data, Table 2 also gives the mean and standard deviation for each stock over all clones. There were differences in mercury tolerance among clones from the same stock. The comparisons between the variances of the stocks indicate, however, that the variability inside the stocks is homogeneous: the comparison between Stocks $\mathrm{PB}_{1}$ and $\mathrm{G}_{\mathrm{I}}$ (which showed the highest and lowest variance, respectively) indicates that no significant difference exists $\left(\mathrm{F}_{3,2}=\right.$ 4.69, $\mathrm{P}>0.05$ ). The data of Table 2 also indicate that there was variability in the stock-averaged median tolerance limits. The results of the one-way analysis of variance indicates that such variability is highly significant $\left(F_{413}=151.34, \mathrm{P}<0.01\right)$. Tukey's test (Lison, 1961) was used to determine where significant differences between stocks occurred. The results of statistical comparisons indicate that there is no difference in tolerance between the $A^{+}$Stocks $G_{1}$ and $G_{V 1 r}$ (from inbreeding populations) but highly significant differences $(P<0.01)$ between these stocks and the $A^{-}$ Stocks $S T, G_{11}$, and $\mathrm{PB}_{1}$ (from outbreeding populations), which were about twice as tolerant to mercury

Table 2. Euplotes crassus. Mercury tolerance $\left(\mathrm{LC}_{50}\right.$ in $\mu \mathrm{M} \mathrm{Hg}^{++}$) of the clones bioassayed for each stock. Stocks designated by different symbols demonstrate significant differences in mercury tolerance, whereas no significant differences occur between stocks designated by the same symbol. One $\mu \mathrm{M} \mathrm{l}^{-1}$ equals $0.2 \mathrm{mg} \mathrm{l}^{-1} \mathrm{Hg}^{++}$

\begin{tabular}{|c|c|c|c|c|c|}
\hline Clones & ${ }^{\bullet} \mathrm{G}_{\mathrm{I}}$ & ${ }^{\bullet} \mathrm{G}_{\mathrm{VII}}$ & $\begin{array}{c}\text { Stocks } \\
\boldsymbol{\Delta}_{S T}\end{array}$ & $\boldsymbol{u}_{\mathrm{G}_{11}}$ & $\mathbf{P B}_{1}$ \\
\hline 1 & 0.257 & 0.264 & 0.520 & 0.674 & 0.665 \\
\hline 2 & 0.236 & 0.301 & 0.502 & 0.615 & 0.636 \\
\hline 3 & 0.272 & 0.269 & 0.570 & 0.680 & 0.710 \\
\hline 4 & & 0.298 & & 0.630 & 0.621 \\
\hline Mean \pm S.D. & $0.255 \pm 0.018$ & $0.283 \pm 0.020$ & $0.531 \pm 0.035$ & $0.650 \pm 0.032$ & $0.658 \pm 0.039$ \\
\hline
\end{tabular}


as the former. Among $\mathrm{A}^{-}$stocks, species-species differences in mercury tolerance appear to occur. In fact, while no difference in tolerance is detectable between the conspecific Stocks $G_{11}$ and $P_{1}$, statistically significant differences $(\mathrm{P}<0.05)$ exist between these stocks and Stock ST belonging to a different biological species. This could reflect the existence of actual differences in the outbreeding degrees of the two biological species. However, the variability recorded amongst $\mathrm{A}^{-}$stocks does not challenge the overall consistency of the relationship of greater tolerance to mercury in outbreeders.

\section{Cross-Breeding Analysis}

Since gene flow occurred between $\mathrm{A}^{+}$and $\mathrm{A}^{-}$ stocks, I sought to gain insight into the control system of the mercury tolerance differences observed. A cross was made between the low-resistance (1,-r.) A+ Stock $G_{V I I}$ and the high-resistance (h.-r.) $A^{-}$Stock $G_{11}$. These stocks were selected because of their better mating reaction and higher viability rate after conjugation. The newly established clones - one from each stock tested for mercury tolerance were involved in the cross. Their phenotypes and genotypes at the unlinked $m t$ (mating type) and a (autogamy) loci, proved to be the same as the original stocks: $G_{V l l}$, mating type P-y (mty) $\left.m t^{4}\right), \mathrm{A}^{+}\left(\mathrm{a}^{+} / \mathrm{a}^{-}\right) ; \mathrm{G}_{11}$, mating type P-w $\left(m t^{\mathrm{w}} / m t^{\mathrm{w}}\right), \mathrm{A}^{-}$ $\left(a^{-} / a^{-}\right)$. (In Euplotes crassus, the mating type trait is under direct genetic control at one locus with a series of multiple alleles, each allele determining a different mating type. There is complete dominance among the alleles (in our case $m t^{w} \ldots>m t^{y} \ldots>m t^{1}$, see below), so that in each diploid cell carrying a combination of two alleles at a time, only one of them is phenotypically expressed (Heckmann, 1964). The single locus a with a pair of alleles $\left(a^{+}, a^{-}\right)$controls the ability to undergo autogamy; the dominant allele $a^{+}$permits the expression of the autogamy trait (Dini and Luporini, 1980). Forty-five pairs were isolated from the $G_{V n 1} \times G_{11}$ mixture, but only 41 of the possible $90 F_{1}$ exconjugant clones, or $45 \%$, survived. Ten of these $F_{1}$ clones, which grew well with a similar fission rate, were selected and bioassayed during the sexual immaturity period. At variance with the procedure for the parental stocks, the set of consecutive steps in mercury concentration used usually included 3 lower concentrations (specifically: $0.10,0.08$, and $0.06 \mu \mathrm{M}$ mercuric ion) beyond the last step of the series reported in the Materials and Methods section.

As I remarked earlier, homotypic pairing may occur in the mixture between Stocks $\mathrm{G}_{\mathrm{VII}}$ and $\mathrm{G}_{11}$. However, the genetic markers in the two stocks made it possible to distinguish between clones from homotypic and heterotypic pairs. When the $10 \mathrm{~F}_{1}$ clones bioassayed reached sexual maturity, they were analysed for both mating type and autogamy trait. Four clones expressed mating type P-w but no autogamy, whereas the other 6 clones displayed both traits, that is, their phenotype was $\mathrm{P}-\mathrm{w}, \mathrm{A}^{+}$. (The $\mathrm{F}_{1}$ clones not bioassayed showed the same segregation pattern.) On the basis of the known genotypes of the parents, this is prima facie evidence that the allele $m t^{\mathrm{w}}$ occupies a higher rank in the domi-

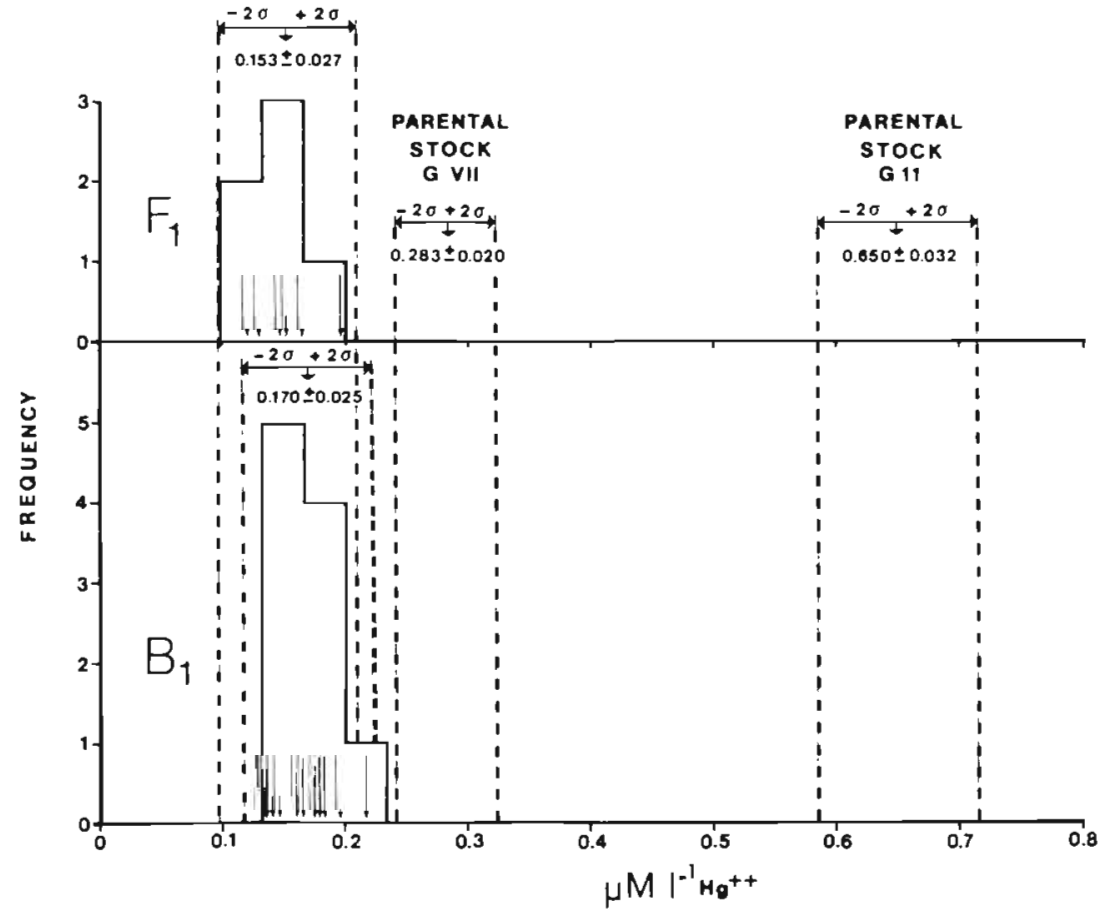

Fig. 1. Euplotes crassus. Frequency distributions of mercury tolerance $\left(\mathrm{LC}_{50}\right.$ in $\left.\mu \mathrm{M} \mathrm{Hg}^{++}\right)$in $F_{1}$ and $\mathrm{B}_{1}$ progenies. Arrows inside histograms indicate 24 - $h$ median tolerance limits of the $F_{1}$ and $B_{1}$ progeny clones bioassayed. In addition, the mean and standard deviation of the clone median tolerance limits for each progeny and parental stock is given. Lines showing $\pm 2 \sigma$ have been drawn to show the approximate limits to the distribution of either progenies or parents 
nance ladder than the $m E$ allele, and suggests that the first 4 clones may be derived from homotypic pairing, whereas the last 6 are surely the outcome of crossfertilization which occurred in heterotypic pairs (from pairing between cells of $G_{\text {VII }}$ and $G_{11}$ ). Accordingly, they had to carry the genotype $m t^{*} / m t^{y}$ or $m t^{w} / m t^{4}, a^{+} /$ $a^{-}$. Only these $6 F_{1}$ clones were further considered. They belonged to 3 synclones, i. e., they derived from 3 pairs in which both partners survived after conjugation. This means that the 2 clones of each synclone were of different cytoplasmic parentage, one deriving from the 1.-r. $G_{V I I}$ the other from h.-r. $G_{11}$. Under these circumstances, one should expect each synclone to show the same parental distinctions when these last are inherited via cytoplasm. The frequency distribution of the 24-h median tolerance limits estimated for the $F_{1}$ clones are reported in Figure 1, along with the mean and standard deviation for the $F_{1}$ over all clones. The $F_{1}$ average median tolerance limit was lower than that of the 1.-r., parental Stock $G_{V I I}$. As shown in Figure $1, F_{1}$ clones of all synclones became alike with respect to mercury tolerance despite their cytoplasmic descent from differentiated parents. Since synclones derived from conjugating partners between which a massive cytoplasmic exchange did not apparently occur, a cytoplasmic inheritance of the trait in question appears inconsequential. Yet, the disappearance of the parental distinctions, and the acquisition of peculiar characteristics by the $F_{1}$ clones in the presence of crossfertilization point towards a significant role of the nucleus in the maintenance of the parental differences in mercury tolerance. Finally, it may be noteworthy that the $4 \quad F_{1}$ clones, which were not considered because they might be from homotypic pairing, behaved similarly to the 6 true hybrid clones with respect to mercury tolerance. This indicates that all the $10 F_{1}$ clones bioassayed were the outcome of crossfertilization.

The mating type uniformity of the $F_{1}$ progeny presented a constraint on further cross-breeding analysis. The results to be presented concern the sole type of cross possible to make, that is, the backcross of a true hybrid $F_{1}$ clone (mating type $\mathrm{P}-\mathrm{w}, \mathrm{A}^{+}$) to the 1.-r., parental Stock $G_{\text {VII }}$ (mating type P-y, $\mathrm{A}^{+}$). Among the true hybrids, the $F_{1}$ clone showing the highest tolerance level was chosen. Owing to the low mating reaction occurring whenever $\mathrm{A}^{+}$animals are involved in the mixtures (Luporini and Dini, 1977), only 28 pairs were isolated and 30 of the possible $56 \mathrm{~B}_{1}$ exconjugant clones, or $53 \%$, survived. All of the $30 \mathrm{~B}_{1}$ progeny clones were retained, but only 10 healthily growing clones with similar fission rates were scored as to mercury tolerance during the sexual immaturity period. There were 3 synclones, plus 4 additional clones whose partners failed to produce a clone. Consi- dered overall, the $B_{1}$ progeny tolerance levels showed a distribution similar to the $F_{1}$; that is, the backcross to the 1.-r., parental Stock $G_{\text {VII }}$ did not result in any tolerance difference with respect to the $F_{1}$ progeny (Fig. 1). After reaching sexual maturity, all the $30 \mathrm{~B}_{1}$ clones retained were analysed for both mating-type and autogamy trait. They fell into 4 phenotypic classes: $\mathrm{P}-\mathrm{w}, \mathrm{A}^{+} ; \mathrm{P}-\mathrm{y}, \mathrm{A}^{+} ; \mathrm{P}-\mathrm{w}, \mathrm{A}^{-} ; \mathrm{P}-\mathrm{y}, \mathrm{A}^{-}$. The respective frequencies observed, 13:8:1:6; are in agreement $\left(\chi^{2}=4.76 ; 0.2>P>0.1\right)$ with a $3: 3: 1: 1$ ratio; two clones were unclassified. Given the genotypes of the Stocks $G_{V I I}$ and $G_{11}$ and the order of dominance of the $m t$ alleles reported above, these are the expected results when a true hybrid $F_{1}$ clone (mating type P-w $\left[m t^{*} /\right.$ $\left.\left.m f^{y}\right], A^{+}\left[a^{+} / a^{-}\right]\right)$is backcrossed with the parental

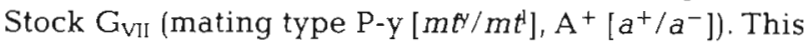
indicates that cross-fertilization had indeed taken place at backcross. Unfortunately, the low mating reactivity and low survival after sexual reorganization precluded using $B_{1}$ clones in further breeding experiments.

In spite of its incompleteness, the cross-breeding analysis is evidence that the mercury tolerance differences existing between the Stocks $A^{+} G_{V \pi}$ and $A^{-} G_{11}$ are (1) determined by nuclear differences, and have (2) no simple genetic basis.

\section{DISCUSSION}

The major conclusion of this study is that non-autogamous $\left(\mathrm{A}^{-}\right)$stocks of Euplotes crassus, representatives of populations following an outbreeding strategy, show a higher resistance to mercury than autogamous $\left(\mathrm{A}^{+}\right)$stocks from inbreeding populations. In this context, consider the results obtained by Persoone and Uyttersprot (1975). These authors investigated the effects of different mercury concentrations on a North European A- multiclonal stock of E. vannus (a typologically-defined species whose separateness from the closely related morphospecies E. crassus is based on historical grounds only; Genermont et al., 1976; Gates, 1978). They found that all specimens survived in $0.1 \mathrm{mg} \mathrm{l}^{-1} \mathrm{Hg}^{++}(=0.50 \mu \mathrm{M})$. This concentration corresponds to the lowest mercury concentration which killed all the test organisms in my $\mathrm{A}^{+}$stocks, whereas it is close to the highest concentration $10.07 \mathrm{mg} \mathrm{l}^{-1}$ $\mathrm{Hg}^{++}=0.32 \mu \mathrm{M}$ ) killing no test organism in the $\mathrm{A}^{-}$ stocks $G_{11}$ and $P B_{1}$. In the light of these considerations, it is a reasonable inference that the $\mathrm{A}^{-}$, multiclonal stock tested by Persoone and Uyttersprot had to show a mercury tolerance level significantly higher than that of $\mathrm{A}^{+}$stocks. Consequently, my conclusion of the relationship of greater tolerance to mercury in $\mathrm{A}^{-}$stocks is strengthened. 
Adaptation to concentrations of metals which are normally toxic is a well-documented phenomenon in various organisms (e.g. the reviews by Ashida, 1965 and Bradshaw, 1971). Among ciliates, Tingle et al. (1973) obtained cytological indications for adaptation to mercuric chloride in the ciliate Tetrahymena pyriformis. It might thus be thought that the higher resistance of the $\mathrm{A}^{-}$stocks of Euplotes is of adaptational origin. Such supposition appears to be unlikely since, except perhaps for the Stock $\mathrm{PB}_{1}$, the other $\mathrm{A}^{-}$ stocks were collected from locations which cannot reasonably be suspected of having high levels of mercuric ion; this is an assumption partially supported by the studies of Piro and Rossi (1979). Furthermore, the $A^{-}$stock $G_{11}$ was collected along the Somalian coasts from the same location as the $\mathrm{A}^{+}$stocks, and it is therefore reasonable to assume that all these stocks were subjected to the same environmental stress.

The results of the cross-breeding analysis suggest that the mercury tolerance differences recorded between the $A^{+} G_{V I l}$ and $A^{-} G_{11}$ stock do not have a simple genetic basis. Two major explanations of the marked decrease in the degree of resistance shown by the $F_{1}$ and $B_{1}$ progenies may be considered. One explanation holds that multiple loci, each having an individual share in the total effect, are controlling mercury tolerance. If this is so, then we would have to assume that (1) the crossed parents were heterozygous at a number of loci, and (2) by chance all the progeny clones tested carried genotypes constituted by combinations of genes specifying low resistance to an extent larger than the low-resistance (l.-r.) Parent $G_{V I I}$. Though a scanty number of progeny clones was examined, the overlapping distribution of the $F_{1}$ and $B_{1}$ tolerances and their sharp downward shift with respect to the 1.-r. parent, render this explanation doubtful.

As it has been stressed previously (Materials and Methods section), there are strong indications of a reduced gene flow between $\mathrm{A}^{+}$and $\mathrm{A}^{-}$stocks which, indeed, follow different ecogenetic strategies; for instance, at variance with $\mathrm{A}^{-}$stocks, $\mathrm{A}^{+}$stocks do not apparently rely on recombinational variety to meet environmental challenges. This calls for an evolutionary divergence and changes in internal genetic organization of the 2 types of stocks. In the light of these arguments a second type of explanation, perhaps more likely, is set forth: The different degrees of susceptibility to mercuric ion shown by the representatives of autogamous (inbreeding) and non-autogamous (outbreeding) populations are the expression of different characteristics of their gene complexes as a whole. Genes in each type of stock form harmonious combinations because they have become coadapted by natural selection. In the hybrids, such coadapted gene complexes are broken up, and a downward shift of mercury tolerance may result. Since the higher resistance of the $\mathrm{A}^{-}$stocks is assumed to be due to a peculiar, internal genetic organization as a consequence of an outbreeding strategy, it may be inferred that their resistance to mercuric ion is only an aspect of a more general property which allows outbreeders to better withstand environmental stress. Indeed, the similar responses to mercury shown by $\mathrm{A}^{-}$stocks of different biological species (Tables 1 and 2), and their common ability to resist lower temperature and $\mathrm{pH}$ than $\mathrm{A}^{+}$stocks (unpubl. observations) provide some support for this argument.

The existence of a relationship between the breeding system of a species and its resistance to environmental stress is not without precedent in the literature. Nyberg (1974) studying different species of freshwater ciliates, belonging to Paramecium aurelia, P. multimicronucleatum, and Tetrahymena pyriformis complexes, noted an impressive consistency of the relationship of greater tolerance to various stressing agents in outbreeders. Nyberg's and the present results both point to a greater ability of outbreeding forms of ciliates to cope directly with changing conditions.

These findings may be used in making a prediction and generalization: environments subjected to sudden changes are expected to be principally colonized by outbreeding forms. The key to the acceptance of the extrapolation to more complicated organisms is (1) that ciliates are facing the same challenges to their survival as are other organisms, and (2) the considerable amount of evidence indicating that ecological responses of Protozoa show many of the same complex phenomena considered to be characteristic of the more advanced organisms.

Acknowledgements. I would like to thank Dr. M. A. Gates (University of Toronto), Dr. P. Luporini (Università di Camerino), and Dr. R. Nobili (Università di Pisa) for reading the manuscript and making helpful suggestions. Assistance and suggestions regarding the statistical elaboration of data were provided by Dr. M. Marchi (Università di Pisa) and Dr. J. J. B. Smith (University of Toronto), to whom I am grateful. I also want to thank Dr. J. Berger (University of Toronto) and Dr. D. Nyberg (University of Illinois) whom I talked to about this problem.

\section{LITERATURE CITED}

Ashida, J. (1965). Adaptation of fungi to metal toxicants. A. Rev. Phytopath. 3: 153-174

Bradshaw, A. D. (1971). Plant evolution in extreme environments. In: Creed, R. (ed.) Ecological genetics and evolution. Blackwell, Oxford, pp. 38-81

Cairns, J., Jr. (1974). Protozoans (Protozoa). In: Fuller, J., Hart, W. (eds) Pollution ecology of freshwater invertebrates. Academic Press, Inc., New York, pp. 1-28 
Dini, F., Bertini, B. (1978). Autogamy-induced effects in aging clones of Euplotes crassus (Ciliata, Hypotrichida). Boll. Zool. 45: 208

Dini, F, Luporini, P. (1980). Genic control of the autogamy trait in the hypotrich ciliate, Euplotes crassus. Genet. Res. 35: $107-119$

Ford, E. B. (1971). Ecological genetics, Chapman and Hall, London.

Gates, M. A. (1978). Morphometric variation in the hypotrich ciliate genus Euplotes. J. Protozool. 25: 338-350

Genermont, J., Machelon, V., Tuffrau, M. (1976). Données expérimentales relatives dans le genre Euplotes (Ciliés Hypotriches). Protistologica 12: 239-248

Glickstein, N. (1979). The potential loss of dissolved mercury and selenium in marine experimentation. Mar. Pollut. Bull. 10: 157

Heckmann, K. (1963). Paarungssystem und genabhängige Paarungstypdifferenzierung bei dem hypotrichen Ciliaten Euplotes vannus O. F. Muller. Arch. Protistenk. 106: $393-421$

Heckmann, K. (1964). Experimentelle Untersuchungen an Euplotes crassus I. Paarungssystem. Konjugation und Determination der Parungstypen. Z. Vererbungsl. 95: $114-124$

Lison, L. (1961). Statistica applicata alla Biologia Sperimentale (Ital. Edit.), Casa Editrice Ambrosiana, Milano

Luporini, P., Dini, F. (1977). The breeding system and the genetic relationship between autogamous and non-autogamous sympatric populations of Euplotes crassus (Dujar- din) (Ciliata Hypotrichida). Monitore zool. ital. (N.S.) 11 119-154

Nanney, D. L. (1980). Experimental Ciliatology: An introduction to genetic and developmental analysis in ciliates, John Wiley \& Sons, New York

Nobili, R. (1967). Induzione di autoconiugazione in mescolanze interspecifiche di Euplotes. (Ciliata Hypotrichida). Arch. Zool. Ital. 52: 51-64

Nyberg, D. (1974). Breeding systems and resistance to environmental stress in ciliates. Evolution 28: 367-380

Nyberg, D. (1975). Genetic analysis of copper resistance in Paramecium aurelia syngen 4. Genetics 80: 463-473

Nyberg, D. (1978). Copper tolerance and segregation distortion in aged Paramecium. Exp. Geront. 13:431-437

Persoone, G., Uyttersprot, G. (1975). The influence of organic and inorganic pollutants on the rate of reproduction of a marine hypotrichous ciliate: Euplotes vannus Muller. Rev. Intern. Océanogr. Méd. 37: 125-151

Piro, A., Rossi, G. (1979). Determinazione di cadmio e mercurio nel Mar Ligure. Atti del Congresso P. F. Oceanografia e Fondi Marini, Roma, pp. 1-13

Sonneborn, T. M. (1957). Breeding systems, reproductive methods, and species problems in protozoa. In: Mayr, E. (ed.) The species Problems. Am. Ass. Advmt Sci. Publ., Washington D.C., pp. 155-324

Tingle, L. E., Pavlat, W. A., Cameron, I. L. (1973). Sublethal cytotoxic effects of mercury chloride on the ciliate Tetrahymena pyriformis. J. Protozool. 20: 301-304 